\title{
Aproximaciones al estudio de las asociaciones como sistemas adaptativos complejos
}

\section{Approaches to the study of associations as Complex Adaptive Systems}

\author{
Luna, Matilde y Cristina Puga (coords.) (20 io), Nuevas \\ PERSPECTIVAS PARA EL ESTUDIO DE LAS ASOCIACIONES, Rubí-ANTHROPOS- \\ Universidad Nacional Autónoma de México, México, 237 Pp., \\ ISBN: 978-84-7658-947-2.
}

En la medida que se ha incrementado el interés por el estudio de la esfera asociativa civil, también ha crecido el interés por evaluar su desempeño. El libro Nuevas perspectivas para el estudio de las asociaciones aborda el dilema, para propósitos de evaluación del desempeño, de efectuar distinciones entre la diversidad de las formas asociativas, a partir de trazar una línea entre los extremos de mayor o menor complejidad que articulan las relaciones en esas organizaciones y el tipo de asociaciones que resultan de esas relaciones. El libro está integrado por nueve ensayos de investigadores que ya cuentan con una trayectoria reconocida en el estudio de esta temática, y se puede describir como un conjunto de propuestas analíticas afortunadas, porque no sólo están integradas entre sí, sino que son innovadoras teórica y metodológicamente. Así, este texto, surgido de un proyecto colectivo de investigación, tiene un carácter multidisciplinario, ya que integra perspectivas trazadas a partir de la política, la sociología y la teoría de la organización, cuyos enfoques consiguen dar a la obra una visión bastante completa de este elusivo objeto de estudio que son las asociaciones. Los temas seleccionados son reveladores de la complejidad del estudio y están unidos por el propósito común de establecer presupuestos válidos y teóricamente sustentados para aproximarse a la evaluación del desempeño de las asociaciones que actúan en los espacios públicos.

La reflexión sobre las asociaciones es, sin lugar a dudas, importante en el mundo contemporáneo por el papel que desempeñan en la economía, la política y el espacio social. La acción de los grupos civiles forma parte de la experiencia cotidiana de atención a los problemas sociales, y el sector solidario se ha ido posicionando como un componente importante del desarrollo. Las fundaciones, las empresas sociales y las organizaciones que promueven los derechos humanos procuran el bienestar y/o la sus- 
tentabilidad del medio ambiente y combaten la pobreza, así como las instituciones asistenciales y otras muy diversas en general son apreciadas positivamente y, en conjunto, se les identifica como un componente del orden que articula las sociedades modernas. En ese contexto, la construcción de modelos de evaluación de su desempeño forma parte de la necesidad de conocer su contribución real al bienestar y de estimar con mayor confiabilidad las ventajas o desventajas de su intervención en los asuntos públicos.

El libro también reviste interés especial para la academia, porque está inserto en una nueva etapa de la investigación sobre el conocimiento de la sociedad civil, ese ámbito disciplinario que podemos calificar como un campo académico emergente y en proceso de consolidación. En nuestro país la investigación académica comenzó a tomar cuerpo en ese campo disciplinario en las décadas de los ochenta y los noventa; cuando comienzan a realizarse los primeros estudios sobre el desarrollo y características de las organizaciones no gubernamentales y del sector no lucrativo, investigación que se amplía y consolida ya en la primera década del siglo xxI. Muestra del interés académico por las asociaciones fue el surgimiento de programas e instituciones enfocadas en esos temas de estudio, así como el levantamiento de encuestas que permitieran registrar y caracterizar a las organizaciones civiles mexicanas. En esa secuencia se identifican: la constitución del Centro Mexicano para la Filantropía (Cemefi), fundado en 1988; la realización de una serie de investigaciones acerca del sector no lucrativo en México y sobre organizaciones civiles y no gubernamentales (Torres, 1998; Méndez, 1998; Verduzco, 2000, 2001, 2003; Pliego, 2001; Calvillo y Favela, 2004); el Programa de Estudios sobre el Tercer Sector, con sede en El Colegio Mexiquense, fundado en 1999; el proyecto Civicus para la construcción de un índice de la sociedad civil en México; la primera Encuesta Nacional sobre Filantropía y Sociedad Civil (Enafi) levantada en 2005; la Encuesta Nacional de Solidaridad y Acción Voluntaria (ENSAV) levantada entre 2005 y 2006, cuyos resultados se publicaron recientemente (Layton, 2006; Verduzco; 2008).

A estos análisis se agregaron los estudios sobre la naturaleza de la sociedad civil, sobre su participación en la esfera pública y en la democracia de América Latina y de México; así como las aportaciones sobre el desarrollo de distintos movimientos sociales y de las redes de organizaciones civiles que han incidido en la vida pública y en la democratización del país (Olvera, 1999, 2001, 2003, 2004; Reygadas, 1988; Cadena 2004; Puga, 2005). Los pasos dados en esa dirección permitieron sumar al conocimiento de las particularidades del sector solidario, el de sus diferencias con otros países, así como identificar e interpretar algunos determinantes de la cooperación entre este sector y el sector público. 
Otras líneas de investigación se dirigieron a examinar los rasgos de la acción colectiva; la orientación, diversidad y desempeño de las organizaciones (Puga y Luna, 2008); la cohesión, la confianza y el capital social (Gordon, 2006; Gordon y Millán, 2009). Paralelamente, el estudio de las formas asociativas se amplió a sus vínculos con el espacio político, con el ámbito empresarial y a su inserción en redes internacionales (Cadena, 2004; Serna, 2008), sin dejar de examinar la transparencia, confianza, rendición de cuentas y efecto en la sociedad (Hernández, 2006; Natal, 2008; Guadarrama, 2008). Podemos entonces decir que la investigación pasó de ser descriptiva a intentar construir los conceptos fundamentales para su quehacer, y que actualmente se encuentra inmersa en construir explicaciones sobre la naturaleza de los procesos asociativos, sus especificidades y diferencias, así como en los mecanismos de cohesión, confianza, solidaridad y cooperación. Las etapas por las que ha transitado esa búsqueda del conocimiento encuentran su explicación en la complejidad y la diversidad de los fenómenos asociativos, en las dificultades para evaluarlos y en la empresa, a veces casi imposible, de recopilar datos y de construir indicadores validos y confiables, obstáculos que desalientan a muchos investigadores.

En resumen, diremos que la amplitud, diversidad y profundidad de la investigación académica realizada en México registra las transformaciones del sector y muestra la importancia que ha adquirido la conformación del campo de estudios que tiene como centro el ámbito de la sociedad civil, y que en ese contexto este libro representa una contribución significativa, porque efectivamente marca nuevas perspectivas para el estudio de las asociaciones.

El carácter innovador de la propuesta analítica estriba en que contempla una forma diferente de examinar las asociaciones, a partir del supuesto de que una parte de ese conjunto funciona como sistemas adaptativos complejos. Una acepción común en el estudio de las asociaciones era considerarlas como unidades operativas simples y relativamente iguales. En la visión general que nos aporta este libro, pero especialmente en el trabajo de Matilde Luna y José Luis Velasco, se plantea que existe un subconjunto en el que las asociaciones pueden ser examinadas como sistemas adaptativos complejos. Es decir, que existen asociaciones que se componen de un gran número de elementos activos cuyos ricos patrones de interacción producen propiedades emergentes que no son fáciles de predecir. Estas asociaciones se perciben como un conjunto de ámbitos y actores que interactúan y se adaptan mediante modificaciones dinámicas. La contribución de esta óptica estriba en que abre la puerta para desarrollar muchos de los conceptos necesarios para examinar la complejidad en las entidades que componen el universo asociativo, ya que no existe to- 
davía una teoría general que proporcione una explicación congruente para esos procesos de interacción.

Otra particularidad del libro está en que sus apartados se encuentran unidos por líneas orientadas a abordar el conjunto de problemas vinculados al propósito común de elaborar un protocolo de evaluación de las asociaciones que considere ángulos que no habían sido abordados, enriqueciéndolos con nuevos y más precisos indicadores. Por ejemplo: 1) en nuestro país no se han desarrollado estudios que consideren, que para examinar las asociaciones, hay que distinguirlas en sus propiedades atendiendo a su composición como sistemas en una línea continua de mayor o menor complejidad; 2) tampoco se había planteado que para evaluar las organizaciones era pertinente contemplar aspectos como la forma en la que construyen sus consensos, toman sus decisiones, establecen sus liderazgos; 3) se agrega una nueva visión al examen del desempeño asociativo al considerar la forma en que las asociaciones se insertan, ejercen influencia, son influidas y, no pocas veces, son condicionadas por su entorno, y 4) al examinar la forma en que utilizan y son interdependientes para procurarse los recursos necesarios.

Esos novedosos enfoques enriquecen la visión sobre el desempeño asociativo de diversas maneras: la incorporación de la toma de decisiones como posible variable en la estimación del desempeño asociativo que construyen Matilde Luna y José Luis Velasco, da paso a la posibilidad de construir indicadores en dimensiones como la autonomía y la confianza. En el mismo sentido, la reflexión sobre el liderazgo como componente o variable del desempeño que realiza Sara Gordon permite explorar cuáles son las cualidades de esa función que favorecen u obstaculizan el desempeño asociativo. En otras miradas dirigidas a los vínculos entre las asociaciones y su entorno, Jorge Cadena y Alejandro Natal abren la ventana que posibilita ver a las asociaciones como sistemas abiertos que interactúan con otros sistemas y, en consecuencia, proponen incluir para la evaluación de su desempeño las interacciones e interdependencias que se establecen. Finalmente, Carlos Chávez Becker nos advierte sobre la pertinencia, en la intención de evaluar la relevancia de las asociaciones, de considerar la medida en que contribuyen a la gobernanza; es un criterio que, no sin cuestionamientos, plantea la posibilidad de establecer indicadores sobre reciprocidad, cultura asociativa, inclusión y exclusión.

Para su lectura resulta muy útil que el libro inicie con las definiciones conceptuales que efectúa Ricardo Tirado sobre el concepto de asociación, las perspectivas que la ciencia social ha desarrollado sobre el tema y las intenciones de construir una tipología de las mismas. Otro apartado sustantivo de la primera parte del volumen es el de Cristina Puga, quien desglosa y construye los rasgos básicos de la propuesta común sobre el 
desempeño asociativo; así, es posible saber de inicio en qué terrenos se mueve este estudio.

Un tanto fuera de las líneas generales, pero igualmente interesantes, son los paseos por los vínculos entre asociaciones y representación en el sistema político que, como Juan Martín Sánchez expresa, ayudan a comprender el creciente papel que tienen las asociaciones en la representación política y, paralelamente, la importancia constitutiva de la representación en las trayectorias asociativas. También invita a la discusión, la reflexión sobre la conveniencia o inconveniencia de la transferencia funcional de modelos desde la esfera empresarial privada hacia los sectores público y solidario, contenida en el ensayo de Montaño Irose.

Se valora como una contribución adicional el hecho de que la reflexión conceptual y metodológica nos sea entregada en un texto ordenado y claro. Primero, porque no es frecuente que los investigadores nos muestren sus dudas y los recorridos epistemológicos que realizan; tampoco es común que presenten al lector los problemas y las soluciones que encuentran para llegar a la construcción de conceptos, líneas e instrumentos de investigación. Segundo, porque el libro no es un patchwork de trabajos aislados, se aprecia como el resultado articulado de un análisis sistemático que arriba a conclusiones y propuestas. Presumiblemente, la propuesta de este grupo de investigadores abrirá la discusión sobre las posibilidades de aplicar sus presupuestos, sobre todo, por las dificultades que implica la operacionalización de algunos de los criterios seleccionados como indicadores de desempeño, pero también por la visión un tanto inclinada a estándares ideales sobre el rol y el funcionamiento de las asociaciones.

\section{Bibliografía}

Cadena-Roa, Jorge (2004), Las organizaciones civiles mexicanas hoy, Universidad Nacional Autónoma de México, México.

Cadena-Roa, Jorge y Cristina Puga (2005) "Criterios para la evaluación del desempeño de las asociaciones", Revista Mexicana de Ciencias Políticas y Sociales, XLVII (193), Universidad Nacional Autónoma de México, México, pp. 13-40,

Calvillo, Miriam y Alejandro Favela Gavia (2004), "Dimensiones cuantitativas de las organizaciones civiles", en Jorge Cadena-Roa (coord.), Las organizaciones civiles mexicanas hoy, Universidad Nacional Autónoma de México, México, pp. 73-120. 
Gordon, Sara y René Millán (2009), Análisis preliminar de la cohesión social. Un estudio comparativo, Universidad Nacional Autónoma de México, México.

Gordon, Sara (2006), “Confianza, reciprocidad y asociatividad: relación indispensable para el desempeño institucional", Estudios Sociológicos, xxIv (71), El Colegio de México, México, pp. 397-422.

Guadarrama-Sánchez, Gloria (2008), "Capacidades de rendición de cuentas de las instituciones de asistencia privada”, en Henio Millán y Alejandro Natal Martínez (coords.), Rendición de cuentas, democracia y sociedad civil en México, El Colegio Mexiquense, Zinacantepec, pp. 175-222.

Hernández-Baqueiro, Alberto (coord.) (2006), Transparencia, rendición de cuentas y construcción de confianza en la sociedad y el Estado mexicanos, Instituto de Acceso a la Información Pública-Centro Mexicano para la Filantropía, México.

Layton D., Michael (2006), "La generosidad en México: ¿quién da? ¿a quién? Discusión de los resultados de la Encuesta Nacional sobre Filantropía y Sociedad Civil (Enafi)", en VIII Encuentro Iberoamericano del Tercer Sector en México, 16-18 de octubre de 2006, Instituto Tecnológico Autónomo de México, México.

Méndez, José Luis, Organizaciones civiles y politicas públicas en México y Centroamérica, Miguel Ángel Porrúa-Academia Mexicana de Investigación en Políticas Públicas, México.

Natal, Alejandro (2008), "El enfoque participativo en proyectos de desarrollo: de lo naive a lo retórico en la cooperación internacional”, en Markus Gottsbacher y S. Lucatello, Reflexiones sobre la ética y la cooperación internacional para el desarrollo: los retos del siglo XXI, Instituto Mora, México, pp. 309-364.

Olvera J., Alberto (1999), La sociedad civil: de la teoría a la realidad, El Colegio de México, México.

Olvera J., Alberto (2001), Movimientos sociales prodemocráticos y esfera pública en México. Sociedad civil y gobernabilidad en México, Universidad Veracruzana, México. 
Olvera J., Alberto (coord.) (2003), Sociedad civil, esfera pública y democratización en América Latina: México, Universidad VeracruzanaFondo de Cultura Económica, México.

Olvera J., Alberto (2004), "Representaciones e ideologías de los organismos civiles en México: crítica de la selectividad y rescate del sentido de la idea de sociedad civil”, en J. Cadena Roa, Las organizaciones civiles mexicanas hoy, Universidad Nacional Autónoma de México, México, pp. 23-47.

Pliego-Carrasco, Fernando (2001), Panorama de las organizaciones no gubernamentales en la Ciudad de México: Encuesta 1997 a organizaciones civiles que asisten, promueven o defienden derechos sociales y ciudadanos, Universidad Nacional Autónoma de México, México.

Puga, Cristina y Matilde Luna (coords.) (2008), Acción colectiva y organización: estudios sobre el desempeño asociativo, Universidad $\mathrm{Na}-$ cional Autónoma de México, México.

Reygada-Robles Gil, Rafael (1988), Abriendo veredas, iniciativas públicas $y$ sociales de las redes de organizaciones sociales, Convergencia de Organismos Civiles por la Democracia, México.

Serna, María Guadalupe (2008), "Organizaciones no lucrativas en México. Estudios de caso", en Jacqueline Butcher (ed.), México solidario. Participación ciudadana y voluntariado, Limusa-Centro Mexicano para la Filantropía, México, pp. 169-194.

Torres, Blanca (1998), "Las organizaciones no gubernamentales: avances de investigación sobre sus características y actuación”, en José Luis Méndez (coord.), Organizaciones civiles y politicas públicas en México y Centroamérica, Miguel Ángel Porrúa-Academia Mexicana de Investigación en Políticas Públicas, México.

Verduzco-Igartúa, Gustavo (2000), Perfil del sector no lucrativo en México, Centro Mexicano para la Filantropía-Universidad Johns Hopkins, México.

Verduzco-Igartúa, Gustavo (2001), "La evolución del tercer sector en México y el problema de su significado en la relación entre lo público y lo privado", Estudios Sociológicos, XIX (55), El Colegio de México, pp. 27-48. 
Verduzco-Igartúa, Gustavo (2003), Organizaciones no lucrativas: visión de su trayectoria en México, El Colegio de México-Centro Mexicano para la Filantropía, México.

Verduzco-Igartúa, Gustavo (2008), "Las acciones solidarias en México (análisis de la Encuesta Nacional de Solidaridad y Acción Voluntaria)", en Jacqueline Butcher (ed.), México solidario: participación ciudadana y voluntariado, Limusa-Centro Mexicano para la Filantropía, México, pp. 57-101.

Recibida: 16 de noviembre de 2010. Aceptada: 30 de noviembre de 2010.

\author{
Gloria J. Guadarrama Sánchez \\ El Colegio Mexiquense, A.C. \\ Correo-e: gguadarr@cmq.edu.mx
}

Gloria J. Guadarrama-Sánchez. Es doctora en ciencias políticas y sociales por la Universidad Nacional Autónoma de México y miembro del Sistema Nacional de Investigadores (SNI). Actualmente es profesora-investigadora en El Colegio Mexiquense, institución en la cual también se ha desempeñado como coordinadora de investigación académica. Sus líneas de investigación son: instituciones, políticas públicas, sociedad civil y tercer sector. Desde 2008 es miembro de la Red de Estudios sobre Desempeño Asociativo (REDA), que agrupa a investigadores e instituciones interesadas en el estudio de las asociaciones civiles. Ha sido docente de los programas de maestría y doctorado en El Colegio Mexiquense, en la Universidad Autónoma del Estado de México y en el Instituto Superior de Ciencias de la Educación del Estado de México. Entre sus publicaciones recientes se cuentan: "La difícil reivindicación de las mujeres. Características del proceso de institucionalización del enfoque de género. El Consejo Estatal de la Mujer y Bienestar Social en el Estado de México", en Emma Liliana Navarrete (coord.), Mujeres mexiquenses. Pasado y presente de las voluntades que transforman, Gobierno del Estado de México, Toluca, pp. 339-378 (2009); "Filantropía y cohesión social: las instituciones asistenciales en el Estado de México", en Jorge Villalobos (coord.), Filantropía y acción solidaria en la historia de México, Centro Mexicano para la Filantropía, pp. 229-264 (2010). 